\title{
The Cochrane HPV vaccine review was incomplete and ignored important evidence of bias
}

\section{Lars Jørgensen $\quad\left[,^{1}\right.$ Peter C Gøtzsche, ${ }^{1}$ Tom Jefferson ${ }^{2}$}

10.1136/bmjebm-2018-111012

${ }^{1}$ Nordic Cochrane Centre, Rigshospitalet (dept. 7811), Copenhagen, Hovedstaden, Denmark

${ }^{2}$ Centre for Evidence Based

Medicine, University of Oxford Centre for Evidence-Based Medicine, Oxford, UK

Correspondence to: Dr Lars Jørgensen, Nordic Cochrane Centre, Copenhagen 2200, Denmark; lj@cochrane. $\mathrm{dk}$

Check for updates

( $)$ Author(s) (or their employer(s)) 2020. No commercial re-use. See rights and permissions. Published by BMJ.

To cite: Jørgensen L, Gøtzsche PC, Jefferson T. BMJ Evidence-

Based Medicine

2020;23:165-168.

\section{Introduction}

The Cochrane Review conducted trial searches up until June 2017 and included 26 randomised trials with 73428 women. ${ }^{1}$ In January 2018, we published an index of the study programmes of the HPV vaccines that included 206 comparative studies. ${ }^{2}$ As of June 2017, about a third of the 206 studies were not published and half of the completed studies listed on ClinicalTrials.gov had no results posted. ${ }^{2}$ Although we sent our index to the Cochrane group handling the Cochrane Review, the review stated that, "nearly all end-of-study reports have been published in the peer-reviewed literature'. When we applied the Cochrane Review's inclusion criteria to the 206 studies, we identified 46 completed and eligible trials. The number of randomised participants could be assessed for 42 of the 46 trials and was 121704 . With nearly half of the trials and half of the participants missing, the Cochrane authors' conclusion, 'that the risk of reporting bias may be small', was inappropriate. Fifteen of the 20 additional trials were listed on ClinicalTrials.gov; the Cochrane authors would therefore have identified more trials if they had searched ClinicalTrials.gov in more depth and searched additional trial registers (we searched 45 trial registers ${ }^{2}$ ).

The Cochrane authors stated that they 'did not include the nine-valent vaccine [Gardasil 9] ... since the randomised trials ... did not incorporate an arm with a non-HPV vaccine control'. This is not correct. The only saline placebo trial of approved HPV vaccines is a Gardasil 9 trial (V503006; NCT01047345) that was published in 2015. ${ }^{3}$ Its participants had previously been vaccinated with four-valent Gardasil, but according to the Cochrane Review protocol, ${ }^{4}$ this was not an exclusion criterion. Since many countries are shifting to Gardasil 9, ${ }^{5}$ it is unfortunate that the Gardasil 9 trial was not included in the Cochrane Review.

\section{No included trial in the Cochrane Review used a placebo comparator}

All 26 trials included in the Cochrane Review used active comparators: adjuvants (aluminium hydroxide $\left(\mathrm{Al}(\mathrm{OH})_{3}\right)$ or amorphous aluminium hydroxyphosphate sulphate) or hepatitis vaccines.

Adjuvants are not regulated separately from their vaccine antigens. According to the Food and Drug Administration, adjuvants are unreliable comparators. $^{6}$ One HPV vaccine manufacturer (GlaxoSmithKline that produces Cervarix) states that its aluminium-based comparator induces harms: 'higher incidences of myalgia might namely be attributable to the higher content of aluminium in the HPV vaccine [450 micrograms $\mathrm{Al}(\mathrm{OH})_{3}$ ] than the content of aluminium in the HAV (hepatitis A) vaccine $\left[225 \text { micrograms } \mathrm{Al}(\mathrm{OH})_{3}\right]^{, 7}$ The comparator hepatitis vaccines also used the HPV vaccines' aluminium-based adjuvant.

The Cochrane authors mistakenly used the term placebo to describe the active comparators. They acknowledged that 'The comparison of the risks of adverse events was compromised by the use of different products (adjuvants and hepatitis vaccines) administered to participants in the control group'. Nevertheless, this statement can easily be overlooked, as it comes after 7500 words about other issues in the discussion and under the heading 'Potential biases in the review process'. Active comparators was not a bias in the review process but a bias in the design of the HPV vaccine trials.

The use of active comparators probably increased the occurrence of harms in the comparator groups and thereby masked harms caused by the HPV vaccines. It is noteworthy that many women were excluded from the trials if they had received the adjuvants before or had a history of immunological or nervous system disorders; for example, in the PATRICIA Trial with 18644 women $^{8}$ and the FUTURE II trial with 12167 women. ${ }^{9}$ These exclusion criteria lowered the external validity of the trials and suggest that the vaccine manufacturers were worried about harms caused by the adjuvants. The criteria are not listed as warnings on the package inserts of the HPV vaccines, ${ }^{10-12}$ which may have led to more vaccine-related harms in clinical practice than in the trials.

\section{The included HPV vaccine trials used composite surrogate outcomes for cervical cancer}

In line with WHO recommendations, ${ }^{13}$ the Cochrane Review was based on composite surrogate outcomes: 'cervical intraepithelial neoplasia grade two and above [CIN2 ${ }^{+}$, CIN grade three and above $\left[\mathrm{CIN}^{+}\right]$, and adenocarcinoma-in-situ (AIS). ${ }^{1}$ The use of such outcomes seemed reasonable for a preliminary assessment of HPV vaccine benefits, but the outcomes can be difficult to interpret. If there were clinically important differences in the severity of the cervical lesions in the two compared groups, they may not have been apparent in the composite outcomes of $\mathrm{CIN}^{+}$and $\mathrm{CIN}^{+}$. The Cochrane authors did not describe any cervical cancers in the 26 trials, although cancers 
did occur in the trials; for example, in the ClinicalTrials.gov entry for the VIVIANE Trial, one case of 'Adenocarcinoma of the cervix' and one case of 'Cervix cancer metastatic' are listed in the HPV vaccine group (see 'Results: Serious Adverse Events'). ${ }^{14}$ Furthermore, the relationship between CIN2 and cervical cancer is not clear-cut. Most CIN2 lesions in women below age 30 years regress spontaneously; an active surveillance approach has therefore been recommended for this group. ${ }^{15}$ The Cochrane Review's 26 trials mainly included women below age 30 years and used frequent cervical screening (often every 6 months) that did not reflect real life practice (often every $3-5$ years ${ }^{5}$ ).

\section{The Cochrane Review incompletely assessed serious and systemic adverse events}

The Cochrane authors reported that they made a 'Particular effort' to assess serious adverse events and performed a sensitivity analysis that gave them "confidence that published and registry or website-sourced data are similar for the same study. ${ }^{1}$ This seems unlikely. As an example, the PATRICIA Trial publication only included two-thirds (1400/2028) of the serious adverse events listed on ClinicalTrials.gov. The Cochrane authors included 701 vs 699 serious adverse events (1400) from the PATRICIA Trial publication (see the Cochrane Reviews' 'Figure 10, Analysis 7.6.2') and 835 vs 829 serious adverse events from its ClinicalTrials.gov entry (see 'Comparison 7, Analysis 6: 7.6.2'; both analyses were called '7.6.2'). We found 1046 vs 982 serious adverse events (2028) when we summarised the data from ClinicalTrials.gov (see 'Results: Serious Adverse Events'). ${ }^{16}$

The Cochrane authors concluded with 'high certainty' that the risk of serious adverse events was similar in the HPV vaccine groups and the comparator groups. However, the authors failed to mention that several of the included trials did not report serious adverse events for the whole trial period. For example, FUTURE $\mathrm{I}^{17}{ }^{17}$ FUTURE $\mathrm{II}^{9}$ and FUTURE III $^{18}$-which in total included 21441 women with up to 4 years follow-up-only reported serious adverse events occurring within 14 days postvaccination. Furthermore, the Cochrane authors did not explain what the serious adverse events consisted of or whether some of them were more common in the HPV vaccine groups.

The Cochrane authors found more deaths in the HPV vaccine groups than in the comparator groups. The death rate was significantly increased in women older than 25 years (risk ratio (RR) 2.36, 95\% CI 1.10 to 5.03; no absolute numbers were provided for this subgroup analysis, but the total numbers of deaths were 51 in the HPV vaccine groups and 39 in the comparator groups). The Cochrane authors suggested that this was a chance occurrence since there was no pattern in the causes of death or in the time between vaccine administration and date of death. However, as the Cochrane Review only included randomised trials, the authors cannot rule out that the increase could be caused by the HPV vaccines. A death may be coded in a way that does not raise suspicion that the vaccine caused it; for example, a 'traumatic head injury' or 'drowning' could have been caused by a 'syncope,' which is a recognised harm..$^{10-12}$ As of May 2018, WHO's pharmacovigilance database-VigiBase, managed by the Uppsala Monitoring Centre (UMC)-contained 499 deaths reported as related to HPV vaccination. ${ }^{19}$

The Cochrane authors concluded that, 'Systemic events with general mild symptoms were similarly frequent in vaccinated recipients and placebo or control vaccine recipients'. Their Analysis 7.5 showed a non-significant increase in systemic events: RR 1.02 (95\% CI 0.98 to 1.07) with a total of 9137 vs
9054 events. The Cochrane authors did not include all of their trials that were eligible for systemic events in Analysis 7.5; for example, the PATRICIA Trial was not included. On ClinicalTrials. gov, PATRICIA has 7129 vs 6557 systemic events listed under 'Results: Other Adverse Events (General disorders)', which in itself is a significantly increased risk: RR 1.09 (95\% CI 1.07 to 1.11). ${ }^{16}$

The Cochrane authors 'planned requesting data from data owners, to fill in gaps with available unpublished data', but 'due to constraints in time and other resources' they were unable to do so. ${ }^{1}$ Considering that 7 years passed from the publication of the Cochrane protocol in $2011^{4}$ to the Cochrane Review in $2018,{ }^{1}$ lack of time seems a poor excuse for not trying to obtain unpublished trial documents and data. More importantly, harms cannot be assessed reliably in published trial documents-especially in journal publications of industry funded trials where even serious harms often are missing. ${ }^{20}$ One reason may be the space restrictions that most medical journals have. As an example, the journal publication for the PATRICIA Trial is 14 pages long $^{8}$ while its publicly available corresponding clinical study report is over 7000 pages long; ${ }^{21}$ although it is an interim report that has been shortened. Clinical study reports are usually confidential documents, but they can be requested from the European Medicines Agency (EMA) and ClinicalStudyDataRequest.com.

Despite the mentioned examples of reporting bias, the Cochrane authors judged all trials at low risk of reporting bias (see the Cochrane Review’s 'figure 4: 'Risk of bias' summary').

\section{The Cochrane Review did not assess HPV vaccine-related safety signals}

The Cochrane authors referred to many observational studies in their discussion that found no safety signals of harms associated with the HPV vaccines. ${ }^{1}$ They cited the WHO's Global Advisory Committee on Vaccine Safety that expressed 'concerns about unjustified claims of harms'. The Cochrane authors did not mention a study from 2017 by the WHO UMC that found serious harms following HPV vaccination overlapping with two syndromes: postural orthostatic tachycardia syndrome (POTS) and complex regional pain syndrome (CRPS). ${ }^{22}$ The WHO UMC provided part of the rationale for EMA's investigation of POTS and CRPS in 2016. ${ }^{23}$ As of May 2018, the WHO UMC VigiBase contained 526 cases of POTS and 168 cases of CRPS reported related to HPV vaccination. $^{19}$

The Cochrane authors did not investigate whether the included trial data reported cases of POTS, CRPS or other safety signals. Instead, the authors cited EMA, which concluded that 'No causal relation could be established' between POTS or CRPS and the HPV vaccines. ${ }^{1}$ The EMA's conclusion was based on the HPV vaccine manufacturers' own unverified assessments ${ }^{23}$ that only included half of the eligible trials. ${ }^{2}$ Furthermore, the HPV vaccine manufacturers search strategies for POTS and CRPS were inadequate and led to cases being overlooked. ${ }^{24}$ As an example, in 2014, the Danish Medicines Agency (DMA) asked the HPV vaccine co-manufacturer Sanofi-Pasteur-MSD to search for specific POTS-related symptoms in its database (including dizziness, palpitations, rapid heart rate, tremor, fatigue and fainting). The manufacturer only searched for 'postural dizziness', 'orthostatic intolerance' and 'palpitations and dizziness'. The DMA discovered this because only 3 of 26 Danish reports of POTS showed up in Sanofi's searches. ${ }^{24}$ As another example, EMA identified six possible cases of POTS and CRPS related to Gardasil 9 that Merck had not identified. ${ }^{25}$ 


\section{Industry trial funding and other conflicts of interest}

The Cochrane authors assessed the impact of industry funding 'by meta-regression. No significant effects were observed. ${ }^{1}$ They stated that, 'All but one of the trials was funded by the vaccine manufacturers', which is not correct. According to ClinicalTrials. gov, this particular trial ('CVT' or 'Costa Rica trial') was sponsored by GlaxoSmithKline. ${ }^{26}$ Therefore, all included trials were funded by the HPV vaccine manufacturers and the meta-regression was meaningless.

The Cochrane Collaboration aims to be free from conflicts of interest related to the manufacturers of the reviewed products. ${ }^{27}$ Most of the 14 Cochrane authors on the first published protocol for the Cochrane Review had major conflicts of interest related to the HPV vaccine manufacturers. ${ }^{28}$ The Cochrane Review only has four authors; three of whom had such conflicts of interest a decade ago. The review's first author currently leads EMA's 'post-marketing surveillance of HPV vaccination effects in non-Nordic member states of the European Union', which is funded by Sanofi-Pasteur-MSD that was the co-manufacturer of Gardasil.

\section{Cochrane's public relations of the review were uncritical}

The announcement of the Cochrane Review on Cochrane.org under 'News' included a 'Science Media Centre roundup of thirdparty expert reaction to this review. ${ }^{29}$ Six experts were cited-all from the UK; although the Cochrane Collaboration is an international organisation. Two of the experts had financial conflicts of interest with the HPV vaccine manufactures. A third expert was responsible for vaccinations in Public Health England that promotes the HPV vaccines. The experts highlighted the 'intensive and rigorous Cochrane analysis', 'that the HPV vaccine is the most effective way for young girls to protect themselves against cervical cancer', and that, 'the vaccine causes no serious side-effects'. No expert criticised the review. In our view, this is not balanced and people with conflicts of interest in relation to the manufacturers should not be quoted in relation to a Cochrane review. Richard Smith-the former editor of the British Medical Journal-described medical journals as an extension of the marketing arm of the drug industry. ${ }^{30}$ We are concerned that some observers may see Cochrane Reviews in the same light when Cochrane publishes such public relations messages.

\section{Conclusion}

Part of the Cochrane Collaboration's motto is 'Trusted evidence'. We do not find the Cochrane HPV vaccine review to be 'Trusted evidence', as it was influenced by reporting bias and biassed trial designs. We believe that the Cochrane Review does not meet the standards for Cochrane Reviews or the needs of the citizens or healthcare providers that consult Cochrane Reviews to make 'Informed decisions', which also is part of Cochrane's motto. We recommend that authors of Cochrane Reviews make every effort to identify all trials and their limitations and conduct reviews accordingly.

\section{Twitter Lars Jørgensen @larsjorgensens1}

Acknowledgements The authors thank Karsten Juhl Jørgensen for helpful comments.

Contributors LJ wrote the first draft. LJ, PCG and TJ contributed to the conception, drafting, critical revision for important intellectual content and the final approval of the article.
Funding The authors have not declared a specific grant for this research from any funding agency in the public, commercial or not-for-profit sectors.

Competing interests TJ was a co-recipient of a UK National Institute for Health Research grant (HTA - 10/80/01 Update and amalgamation of two Cochrane Reviews: neuraminidase inhibitors for preventing and treating influenza in healthy adults and children- https://www.journalslibrary.nihr.ac.uk/ programmes/hta/108001\#/). TJ is also in receipt of a Cochrane Methods Innovations Fund grant to develop guidance on the use of regulatory data in Cochrane reviews. TJ is occasionally interviewed by market research companies about phase I or II pharmaceutical products. In 2011-2014, TJ acted as an expert witness in a litigation case related to the antiviral oseltamivir, in two litigation cases on potential vaccine-related damage and in a labour case on influenza vaccines in healthcare workers in Canada. He has acted as a consultant for Roche (1997-1999), GSK (2001-2002), Sanofi-Synthelabo (2003) and IMS Health (2013). In 2014-2016, TJ was a member of three advisory boards for Boehringer Ingelheim. TJ was a member of an independent data monitoring committee for a Sanofi Pasteur clinical trial on an influenza vaccine. TJ and PCG were co-signatories of a complaint to the European Ombudsman on maladminstration in relation to the EMA investigation of possible harms from HPV vaccines. PCG does not regard this as a competing interest.

Patient consent for publication Not required.

Provenance and peer review Not commissioned; externally peer reviewed.

ORCID iD

Lars Jørgensen http://orcid.org/0000-0002-9737-0555

\section{References}

1 Arbyn M, Xu L, Simoens C, et al. Prophylactic vaccination against human papillomaviruses to prevent cervical cancer and its precursors. Cochrane Database Syst Rev 2018;5:CD009069.

2 Jørgensen L, Gøtzsche PC, Jefferson T. Index of the human papillomavirus (HPV) vaccine industry clinical study programmes and non-industry funded studies: a necessary basis to address reporting bias in a systematic review. Syst Rev 2018;7:8.

3 Garland SM, Cheung T-H, McNeill S, et al. Safety and immunogenicity of a 9-valent HPV vaccine in females 12-26 years of age who previously received the quadrivalent HPV vaccine. Vaccine 2015;33:6855-64.

4 Arbyn M, Bryant A, Martin-Hirsch PPL, et al. Prophylactic vaccination against human papillomaviruses to prevent cervical cancer and its precursors. Cochrane Database Syst Rev 2013;38.

5 WHO. Human papillomavirus (HPV) position paper. who, 2017. Available: http://www.who.int/immunization/policy/position_papers/hpv/en/ [Accessed May 2018].

6 Krause P. Update on vaccine regulation: Expediting vaccine development. FDA/CBER/OVRR. Available: https://c.ymcdn.com/sites/www.casss.org/ resource/resmgr/CMC_Euro_Speaker_Slides/2014_CMCE_KrausePhil.pdf [Accessed May 2018].

7 GSK Study Register - Study 104951. Available: https://www.gsk-clinical studyregister.com/study/104951? search=studyEtsearch_terms $=104951$ \#csr [Accessed May 2018].

8 Paavonen J, Naud P, Salmerón J, et al. Efficacy of human papillomavirus (HPV)-16/18 AS04-adjuvanted vaccine against cervical infection and precancer caused by oncogenic HPV types (PATRICIA): final analysis of a double-blind, randomised study in young women. Lancet 2009;374:301-14.

9 FUTURE II Study Group. Quadrivalent vaccine against human papillomavirus to prevent high-grade cervical lesions. N Engl J Med 2007;356:1915-27. 
10 Research C for BE and. Approved Products - Cervarix. Available: https:// www.fda.gov/BiologicsBloodVaccines/Vaccines/ApprovedProducts/ ucm 186957.htm [Accessed May 2018].

11 Research C for BE and. Approved Products - Gardasil. Available: https:// www.fda.gov/BiologicsBloodVaccines/Vaccines/ApprovedProducts/ UCM094042 [Accessed May 2018].

12 Research C for BE and. Approved Products - Gardasil 9. Available: https:// www.fda.gov/BiologicsBloodVaccines/Vaccines/ApprovedProducts/ ucm426445.htm [Accessed May 2018].

13 Pagliusi SR, Teresa Aguado M. Efficacy and other milestones for human papillomavirus vaccine introduction. Vaccine 2004;23:569-78.

14 Study to Evaluate the Efficacy of the Human Papillomavirus Vaccine in Healthy Adult Women of 26 Years of Age and Older - Study Results - ClinicalTrials.gov. Available: https://clinicaltrials.gov/ct2/show/results/ NCT00294047 [Accessed May 2018].

15 Tainio K, Athanasiou A, Tikkinen KA0, et al. Clinical course of untreated cervical intraepithelial neoplasia grade 2 under active surveillance: systematic review and meta-analysis. BMJ 2018;360:k499.

16 ClinicalTrials.gov. Human Papilloma Virus (HPV) Vaccine Efficacy Trial Against Cervical Pre-cancer in Young Adults With GlaxoSmithKline (GSK) Biologicals HPV-16/18 - Study Results. Available: https://clinicaltrials.gov/ ct2/show/results/NCT00122681 [Accessed May 2018].

17 Garland SM, Hernandez-Avila M, Wheeler CM, et al. Quadrivalent vaccine against human papillomavirus to prevent anogenital diseases. $N$ Engl $J$ Med 2007;356:1928-43.

18 Muñoz N, Manalastas R, Pitisuttithum P, et al. Safety, immunogenicity, and efficacy of quadrivalent human papillomavirus (types 6, 11, 16, 18) recombinant vaccine in women aged $24-45$ years: a randomised, doubleblind trial. The Lancet 2009;373:1949-57.

19 VigiAccess. Available: http://www.vigiaccess.org/ [Accessed May 2018].

20 Golder S, Loke YK, Wright K, et al. Reporting of adverse events in published and unpublished studies of health care interventions: a systematic review. PLoS Med 2016;13:e1002127.

21 Interim clinical study report for study 580299/008 (HPV-008). Available: https://www.gsk-clinicalstudyregister.com/files2/gsk-580299-008-clinicalstudy-report-redact.pdf [Accessed May 2018].
22 Chandler RE. Safety concerns with HPV vaccines continue to Linger: are current vaccine pharmacovigilance practices sufficient? Drug Saf 2017;40:1167-70.

23 Jefferson T, Jørgensen L. Human papillomavirus vaccines, complex regional pain syndrome, postural orthostatic tachycardia syndrome, and autonomic dysfunction - a review of the regulatory evidence from the European medicines Agency. Indian J Med Ethics 2017;2.

24 Gøtzsche PC, Jefferson T, Brinth LS, et al. Complaint to the European Ombudsman over maladministration at the European medicines Agency (EMA) in relation to the safety of the HPV vaccines, 2016. Available: http://nordic.cochrane.org/sites/nordic.cochrane.org/files/public/uploads/ ResearchHighlights/Complaint-to-ombudsman-over-EMA.pdf [Accessed May 2018].

25 Rapporteurs' Day 150 Joint Response Assessment Report of Gardasil 9. Procedure no. EMEA/H/C/3852. CHMP 2014.

26 ClinicalTrials.gov. Vaccine To Prevent Cervical Intraepithelial Neoplasia or Cervical Cancer in Younger Healthy Participants - Full Text View. Available: https://clinicaltrials.gov/ct2/show/NCT00128661 [Accessed May 2018].

27 Conflict of interest policy for Cochrane groups | Cochrane community. Available: http://community.cochrane.org/organizational-info/resources/ policies/conflict-interest-policy-cochrane-groups [Accessed May 2018].

28 Schaaber J. Cochrane under influence: assessment of the HPV vaccines and conflict of interest. ISDB Newsletter 2013;28:8.

29 Scientific expert reaction to new cochrane review on HPV vaccine for cervical cancer prevention in girls and women | Cochrane, 2018. Available: http://www.cochrane.org/news/scientific-expert-reaction-new-cochranereview-hpv-vaccine-cervical-cancer-prevention-girls-and [Accessed May 2018]

30 Smith R. Medical journals are an extension of the marketing arm of pharmaceutical companies. PLoS Med 2005;2:e138.

31 Cochrane Handbook for systematic reviews of interventions | Cochrane training. Available: http://training.cochrane.org/handbook [Accessed May 2018]. 


\section{Correction: The Cochrane HPV vaccine review was incomplete and ignored important evidence of bias}

Jørgensen L, Gøtzsche PC, Jefferson T. The Cochrane HPV vaccine review was incomplete and ignored important evidence of bias. BMJ Evidence-Based Med 2018;23:165-8. doi:10.1136/ bmjebm-2018-111012

This article (https://ebm.bmj.com/content/23/5/165) has a correction. The changes are clarificatory, and for this reason, the editors have issued a correction and not a retraction. A linked Editor's note provides more background to this decision, and a marked copy is available to view (online supplementary appendix 1) .

The corrections are outlined below.

An additional table outlining the authors' reassessment of 20 studies identified as additionally eligible for the Cochrane HPV vaccine review has been included in the article (online supplementary table 1). Sixteen additional trials were eligible for inclusion in the Cochrane HPV Review (not 20 as stated in the article). Additional data from four trials already included in the Cochrane HPV Review (NCT00929526; NCT00518336; NCT00652938; NCT00578227) are potentially eligible for inclusion.

The number of randomised participants could be assessed for 42 of the 46 trials, and the authors found an additional 25550 females (and possibly up to 30195 for the Cochrane HPV Review's serious adverse events meta-analyses) who are eligible for the Cochrane HPV Review's meta-analyses. In the analysis, the authors did not originally subtract the male participants that were included in three of the studies.

The PATRICIA trial publication only included two thirds (1400/2028) of the serious adverse events listed on ClinicalTrials.Gov". The PATRICIA trial registry reports the total number of women with serious adverse events within each MedDRA preferred term category, ${ }^{1}$ which yields a different total number of women than the total described in the PATRICIA published report and also in the Cochrane HPV Review. ${ }^{2}$ The NCT entry reports the number of women with serious adverse events for each MedDRA term. The final total number of serious adverse events as a proportion of total events remains unknown.

Industry trial funding and other conflicts of interest:

"The Costa Rican Vaccine Trial is a longstanding collaboration between investigators in Costa Rica and NCI. The trial is sponsored and funded by NCI (N01-CP-11005) with support from the NIH Office of Research on Women's Health and conducted in agreement with the Ministry of Health of Costa Rica." The trial publication reports that the "Vaccine was provided for our trial by GSK (GlaxoSmithKline) Biologicals, under a Clinical Trials Agreement with NCI. GSK also provided support for aspects of the trial associated with regulatory submission needs of the company under FDA (Food and Drug Administration) BB-IND 7920. D R Lowy and J T Schiller are named inventors on the US government-owned HPV vaccine patents that are licensed to GSK and Merck, and so are entitled to limited royalties as specified by federal law.”

In the context of FDA regulations, the trial may have been sponsored by GSK, but it is not clear if the trial received any funding from GSK. We consider it is reasonable to accept that GSK provided funding, at least in some kind, since it provided vaccines and support related to the regulatory submission. Therefore, all included trials were funded or sponsored by the HPV vaccine manufacturers.

( Author(s) (or their employer(s)) 2020. No commercial re-use. See rights and permissions. Published by BMJ.

BMJ Evidence-Based Medicine 2020;25:e3. doi:10.1136/bmjebm-2018-111012corr1

Check for updates 


\section{Editors' Note: “The Cochrane HPV vaccine review was incomplete and ignored important evidence of bias"}

Carl Heneghan, Igho Onakpoya

A Cochrane systematic review of human papillomavirus (HPV) vaccine (hereafter referred to as the Cochrane HPV Review) was published on 9th May 2018.

The article, 'The Cochrane HPV vaccine review was incomplete and ignored important evidence of bias' was submitted to BMJ EBM on 24th May 2018 for the 'Debate, analysis and opinion' section of the journal. The handling editor, Dr Igho Onakpoya (Research Editor, BMJ EBM) sent it for external peer review to an expert in HPV vaccines and for internal peer review to Professor Carl Heneghan, Editor in Chief of BMJ EBM. Peer reviewers' reports were returned by 19th June and were sent to the authors, who were invited to make revisions. The revised version was submitted on third July, accepted by the handling editor on seventh July and published online on 27th July $2018 .{ }^{1}$

The analysis of the Cochrane HPV review stated there were missing eligible trials, reporting bias, and biased trial designs and conflicts of interest. ${ }^{23}$

Cochrane initiated an investigation in response to the criticism and published a response to the article on third September authored by Cochrane's then Editor in Chief (EiC) David Tovey and deputy EiC Karla Soares-Weiser. This response defended the Cochrane HPV Review and outlined key findings from the Cochrane investigation:

- The Cochrane Review did not miss "nearly half of the eligible trials". A small number of studies were missed due to the primary focus on peer-reviewed reports in scientific journals, but the addition of these data makes little or no difference to the results of the review for the main outcomes;

- The trials comparators were unambiguously, transparently, and accurately described;

- The selection of outcomes for benefits was appropriate and was consistent with WHO guidance;

- The review included published and unpublished data on serious harms, and the findings on mortality were reported transparently and responsibly;

- The review was compliant with Cochrane's current conflict of interest policy;

- Cochrane's media coverage was cautious and balanced, but we recognise that there could be improvements in relation to transparency where external experts are quoted;

- The BMJ Evidence-Based Medicine article "substantially overstated its criticisms"

The response also criticised the peer review process of the journal and whether the conclusions were justified and proportionate. When these criticisms were raised with the journal, we embarked on a lengthy clarification process with the authors and Cochrane.

September 2018

We wrote to the authors of the BMJ EBM analysis article and received a response that asserted '(their) analysis was appropriate and that the Cochrane editors substantially ignored several of (their) criticisms. ${ }^{5}$ We also contacted Cochrane's EiC and the Cochrane corresponding author to ask for further details of their criticisms of the BMJ EBM peer review process and how the article was overstated.

We set out the contentious questions arising from the Cochrane Editors' response and considered whether the BMJ EBM analysis ${ }^{6}$ required further editorial comments or corrections. We sent a copy of this review to all parties concerned and posted it on BMJ EBM Spotlight on $16^{\text {th }}$ of October 2018. Our review concluded that the BMJ EBM analysis required several corrections and that the overall article did not overstate its claims, nor did it warrant retraction.

The authors of the BMJ EBM analysis article responded to the conclusions of the Cochrane investigation in a rapid response to the original article. They acknowledged and approved the conclusions of the BMJ EBM Editors' Review and provided further details and clarification.Jørgensen et al. 2018 October 2018

The Cochrane HPV review corresponding author contacted The BMJ to enquire whether The BMJ might provide a forum to reply. The BMJ EiC suggested sending a detailed response to the BMJ EBM publication, a rapid response to The BMJ and potentially an opinion piece about the broader issues. November 2018

Cochrane EiC emailed the BMJ EBM EiC to say that there remained uncertainties with identifying studies for inclusion. Six studies had now been identified and will be added to the Cochrane HPV Review. Their re-analysis of the data incorporating the missing data suggested that their results did not change with the inclusion of this data. The email also reiterated concerns with the BMJ EBM peer review process for that article, specifically that there was only one external peer reviewer. 2019

Since then BMJ EBM has updated its editorial policy to ensure EBM Analysis pieces are reviewed by two external peer reviewers at a minimum. Debate, Insights and opinion pieces are sent to external 
or internal review, and EBM verdicts as commentaries are subject to internal review. The journal's peer review policy is stated on the BMJ EBM website to increase transparency.

We have published a correction to the BMJ EBM HPV analysis article, ${ }^{1}$ alongside this linked Editor's Note.

The BMJ EBM analysis article by Jørgensen, Gøtzsche and Jefferson defined methods for securing the available evidence and we consider the article title, and conclusions, are justified. Until the updated review is published we remain unclear about the actual number of trials that require inclusion in the Cochrane Review, and whether the missing trials impact on the Cochrane results.

Competing interests

$\mathrm{CH}$ has co-authored 16 peer-reviewed articles with Tom Jefferson (two of which are Cochrane reviews) and holds grant funding jointly with Tom Jefferson from Cochrane on methods for deciding when to prioritise the use of clinical study reports in systematic reviews. $\mathrm{CH}$ is a member of Cochrane and a contact editor for the Cochrane Acute Respiratory Infection group and has been an author 21 Cochrane reviews including updates. He is a founder of AllTrials and an advisor to the WHO International Clinical Trial Registry Platform. and He has received expenses and fees for his media work (including payments from BBC Radio 4 Inside Health). He has received expenses from the WHO and holds grant funding from the NIHR, the NIHR School of Primary Care Research, NIHR BRC Oxford and Cochrane. He has received financial remuneration from an asbestos case and given free legal advice on mesh cases. He has also received income from the publication of a series of toolkit books published by Blackwells. On occasion, he receives expenses for teaching EBM and is also paid for his NHS GP work in urgent care (contract with Oxford Health NHS Foundation Trust). He is Director of CEBM at the University of Oxford, which jointly runs the EvidenceLive Conference with the BMJ and the Overdiagnosis Conference with international partners, based on a non-profit making model. He is Editor in Chief of BMJ Evidence-Based Medicine. IO is Research Fellow in Evidence Synthesis at the CEBM, University of Oxford, a clinician who works across several NHS Trusts and is funded by the NIHR School of Primary Care Research. IO has co-authored eight peer-reviewed articles jointly with Tom Jefferson (one of these is a Cochrane review).

\section{REFERENCES}

1. Jørgensen L, Gøtzsche PC, Jefferson T. The Cochrane HPV vaccine review was incomplete and ignored important evidence of bias BMJ evidence-based medicine 2018; 23:165-168. Available: https://ebm.bmj.com/content/23/5/165 [Accessed 3 Sep 2019].

2. Hawkes N. Cochrane editors hit back at criticisms of HPV vaccine review. BMJ 2018;362:k3834. doi: 10.1136/bmj.k3834

3. Arbyn M, Xu L, Simoens C, et al. HPV vaccination to prevent cancer and pre-cancerous changes of the cervix. Cochrane 2018.

4. Tovey D, Soares-Weiser K. Cochrane's Editor in Chief responds to a BMJ Evidence-Based Medicine article criticizing the Cochrane Review of HPV vaccines. Available: https://www.cochrane.org/sites/ default/files/public/uploads/cochrane_hpv_response_3sep 18.pdf

5. Jørgensen L, Jefferson GP. Response to Cochrane editors: Jørgensen, Gøtzsche and Jefferson | BMJ EBM spotlight, 2018. Available: https://blogs.bmj.com/bmjebmspotlight/2018/09/23/responseto-cochrane-editors-jorgensen-gotzsche-and-jefferson/ [Accessed 27 Sep 2018].

6. Jørgensen L, Gøtzsche PC, Jefferson T. The Cochrane HPV vaccine review was incomplete and ignored important evidence of bias. BMJ Evid Based Med 2018;23:165-8. doi:10.1136/bmjebm-2018-111012

๑ Author(s) (or their employer(s)) 2020. No commercial re-use. See rights and permissions. Published by BMJ.

BMJ Evidence-Based Medicine 2020;25:e2. doi:10.1136/bmjebm-2018-111012ednote

Check for updates 